\title{
Pétrole et sécurité privée au Nigeria : un complexe multiforme à l'épreuve du
}

Marc-Antoine Pérouse de Montclos

\section{(2) OpenEdition}

\section{Journals}

Édition électronique

URL : http://journals.openedition.org/conflits/983

DOI : $10.4000 /$ conflits.983

ISSN : $1777-5345$

Éditeur :

CCLS - Centre d'études sur les conflits lilberté et sécurité, L'Harmattan

Édition imprimée

Date de publication : 1 décembre 2003

Pagination : 117-138

ISBN : 2-7475-6065-1

ISSN : 1157-996X

Référence électronique

Marc-Antoine Pérouse de Montclos, «Pétrole et sécurité privée au Nigeria : un complexe multiforme à l'épreuve du », Cultures \& Conflits [En ligne], 52 I hiver 2003, mis en ligne le 03 juillet 2004, consulté le 30 mars 2021. URL : http://journals.openedition.org/conflits/983 ; DOI : https://doi.org/10.4000/ conflits.983

Ce document a été généré automatiquement le 30 mars 2021.

Creative Commons License 


\title{
Pétrole et sécurité privée au Nigeria : un complexe multiforme à l'épreuve du
}

\author{
Marc-Antoine Pérouse de Montclos
}

Depuis la fin de la guerre froide et le triomphe des idées libérales, la privatisation de l'Etat est devenu un thème de prédilection des études sur les pays en développement. Certains ont analysé le phénomène sous l'angle économique en insistant sur le coût social $\mathrm{du}$ démantèlement des services publics qu'entraînaient les programmes d'ajustement de la Banque mondiale. D'autres y ont vu une consécration de la criminalisation de l'Etat, en particulier dans les «kleptocraties» africaines où la personnalisation du pouvoir entretenait une grande confusion des intérêts publics et privés. Dans tous les cas, qu'il s'agisse des secteurs de la santé, de l'eau ou de l'éducation, les processus de privatisation économique ont souvent été perçus comme le signe d'un recul de l'Etat sur le plan politique.

Concernant l'Afrique noire, la théorie des vases communicants, qui établissait une corrélation étroite entre l'affaiblissement de la puissance publique et le renforcement $\mathrm{du}$ domaine privé, a cependant été reconsidérée à l'aune de pratiques qui contredisaient les explications simplistes. Le lien de cause à effet n'était pas évident. De la Somalie au Libéria en passant par le Sud du Soudan, l'Etat africain, d'abord, n'a pas eu besoin de libéralisation économique pour s'effondrer. La vague de privatisations, ensuite, a aussi touché les appareils étatiques les plus solides du continent, à commencer par l'Afrique du Sud. Les mouvances présidentielles, surtout, ont parfaitement su profiter des opportunités qu'offraient les programmes d'ajustement de la Banque mondiale. Au Nigeria ou en Ouganda, des proches du pouvoir ont ainsi racheté à bon prix les firmes publiques vendues à l'encan.

De leur côté, les entreprises privées se sont adaptées aux changements en cours. Les multinationales du pétrole, notamment, ont réussi à investir dans des économies déstructurées, voire mafieuses, en dépit de contextes insurrectionnels qui, du Soudan à l'Angola, les ont obligées à sécuriser leurs opérations avec les moyens du bord. A partir 
d'enclaves abritant leur personnel expatrié, ces compagnies ont, en l'occurrence, employé des gardes privés et loué les services des forces de l'ordre locales. Quand elles ne pouvaient pas délocaliser ou sous-traiter les activités les plus exposées, elles ont également dû «acheter » la complicité des populations alentours et pallier les lacunes d'administrations défaillantes en gérant des projets de développement et en assurant un service public à minima.

Premier producteur d'hydrocarbures en Afrique, le Nigeria constitue un exemple emblématique des complexes " pétro-sécuritaires » de ce type. La collusion des intérêts publics et privés, l'immixtion des militaires dans les milieux d'affaires, un niveau élevé de corruption et une violence endémique ont en effet conduit les multinationales en place à se replier dans des ghettos. Leurs «zones industrielles renforcées " ont alors révélé l'existence d'une sorte de "syndrome de Monaco " consistant à défendre une minorité de privilégiés contre les assauts de la majorité, exclue des bénéfices de la croissance. Dans un tel dispositif, la force publique a eu pour mission de protéger en priorité des intérêts privés : une pratique déjà en usage du temps de la colonisation, bien avant la libéralisation des économies africaines sous la coupe de la Banque mondiale dans les années 1980. Un rapide rappel historique montre ainsi que la privatisation de la sécurité au Nigeria relève moins d'une dynamique conjoncturelle que d'une économie politique dont les fondements sont bien ancrés dans la vie du pays. Une armée d'hommes d'affaires

Depuis l'indépendance en 1960, le Nigeria a connu une impressionnante succession de régimes civils et militaires qui a débouché sur la formation d'une sorte de joint-venture entre l'armée et les milieux d'affaires. Les juntes au pouvoir ont placé des hommes de confiance à la tête des grandes entreprises parapubliques, essentiellement dans les secteurs du pétrole, de l'électricité et des transports aérien ou maritime. Les milieux d'affaires, de leur côté, se sont volontiers associés aux militaires pour profiter du boom pétrolier des années $1970^{1}$. Ni le retour des soldats dans les casernes ni la restauration de régimes parlementaires en 1979, 1993 et 1999 n'ont mis fin à une association aussi lucrative. Enrichis par leur passage au pouvoir, les militaires à la retraite n'ont pas renoncé aux affaires ; au contraire, ils ont pu s'y consacrer à plein temps ${ }^{2}$.

Grâce à leurs accointances, certains ont sous-traité les appels d'offre du ministère de la Défense et monopolisé les approvisionnements de l'armée ${ }^{3}$. Profitant des nationalisations du début des années 1970 puis, en retour, des privatisations de la fin des années 1980, notamment le Nigerian Entreprises Promotion Decree de 1989, d'autres se sont positionnés dans la distribution, l'import-export, la construction, l'immobilier, le pétrole, l'industrie et la banque, voire le transport maritime pour les officiers de la marine. Le "triumvirat " des généraux Obasanjo, Yar'adua et Danjuma, au pouvoir entre 1976 et 1979, est significatif à cet égard. Obasanjo, avec sa ferme de Sango Ota près d'Abéokuta, et Yar'adua, avec les complexes de Sambo Farms à Birnin Gwari, près de Kaduna, et de Mandara Farms, sur le Plateau, se sont lancés dans l'agriculture. Directeur de la Defence Industries Corporation of Nigeria à partir de 1984, Danjuma, lui, s'est reconverti dans des activités à priori peu compatibles avec la fonction militaire, telle l'importation de sel.

Naturellement, les convoitises des officiers supérieurs ont suivi l'évolution de l'économie nigériane, qui reposait sur l'agriculture à l'Indépendance. Outre les prébendes du ministère de la Défense, les caisses de l'État, les organes de gouvernement et les entreprises parapubliques ont constitué les cibles privilégiées de 
la prévarication des militaires. Au sortir de la guerre du Biafra, le régime du colonel Yakubu Gowon s'est d'abord illustré en concédant à ses fidèles la direction des institutions régionales démantelées avec la fédéralisation du pays, telle la Northern Nigerian Development Company, qui avait des intérêts dans l'agroalimentaire, la construction, le logement, et qui fut simplement rebaptisée New Nigerian Development Company pour ne rien changer aux initiales de son sigle. Les militaires du Nord et leurs alliés ont également mis la main sur les principaux établissements financiers où passaient à l'époque les fonds de la Banque mondiale, à savoir la Federal Mortgage Bank et la Nigerian Industrial Development Bank. Dans les années qui suivirent, on a, par exemple, retrouvé le général Shehu Musa Yar'adua à la tête de l'Habib Bank, le capitaine Usman Jibrin à la Nigerian Agricultural and Cooperative Bank, le général Theophilus Danjuma à l'Universal Trust Bank et le sultan de Sokoto, Ibrahim Dasuki, à la présidence de la branche nigériane de la Bank of Credit and Commerce International, la fameuse BCCI.

Mais c'est évidemment dans le secteur pétrolier que les malversations de la classe dirigeante devaient ensuite prendre toute leur ampleur, notamment à travers la NNPC (Nigeria National Petroleum Corporation), inaugurée par le général Olusegun Obasanjo en 1976 et véritable «trou noir » où, encore aujourd'hui, «s'évapore » la majeure partie des revenus du Trésor public. Le pétrole nigérian, qui est exporté à $44 \%$ vers les ÉtatsUnis et à $39 \%$ vers l'Europe, fournit l'essentiel des rentrées en devises du gouvernement. Quand on sait que le pays représente 3\% de la production pétrolière et $2 \%$ des réserves de gaz naturel dans le monde, on imagine l'ampleur des détournements de la rente, qui se chiffrent en milliards de dollars. Outre la NNPC, bien des militaires ont réussi à s'imposer dans le conseil d'administration des succursales nigérianes des multinationales du pétrole, où ils ont servi d'intermédiaires pour faciliter l'obtention de contrats auprès du gouvernement. N'oublions pas non plus les petites compagnies locales qui, profitant de la vague de privatisation de la fin des années 1980, ont réussi, par l'entremise des gradés au pouvoir, à obtenir une ou plusieurs licences d'exploitation de gisements de pétrole, souvent pour le compte des multinationales ou de quelque général reconverti dans les affaires ${ }^{4}$.

Dans le cadre du programme d'ajustement structurel adopté sous l'égide de la Banque mondiale à partir de 1986, la libéralisation de l'économie n'a certainement pas gêné les militaires qui, à titre privé, ont racheté à bon prix les entreprises publiques qu'ils avaient nationalisées avec les « décrets d'indigénisation » du début des années 1970. Au pouvoir entre 1985 et 1993, la junte du général Ibrahim Babangida est passée maître dans l'art de revendre des faveurs ou d'empocher des commissions au moment de la signature d'un contrat. Son successeur, le général Sani Abacha, n'a pas démérité. Il a détourné une partie de la rente pétrolière, placée sur des comptes offshore à l'étranger, et a réalisé de substantiels bénéfices lorsque, début 1995, les autorités ont mis fin au contrôle des changes tout en autorisant les organismes étatiques à continuer d'appliquer le taux officiel de 22 naira pour un dollar américain, qui était quatre fois inférieur au cours du marché. De hauts responsables ont, pour leur part, empoché les émoluments de salariés fantômes. On estime qu'entre 1997 et 2001, le gouvernement fédéral a payé plus de trente milliards de naira à des employés qui n'existaient pas ${ }^{5}$ !

Le pétrole, ou l'huile sur le feu

Bien entendu, le développement du pays s'en est ressenti d'autant et la frustration des laissés pour compte du boom pétrolier a fini par éclater au grand jour. A la différence d'autres pays producteurs d'hydrocarbures comme le Soudan ou l'Angola, le Nigeria 
n'est pas officiellement en guerre. Mais le delta du Niger, où l'on extrait de l'or noir depuis une cinquantaine d'années, connaît un très fort taux de violence criminelle ainsi qu'un état de rébellion endémique. L'aire culturelle ijaw, notamment, compte aujourd'hui parmi les plus affectées par l'agitation contre les compagnies pétrolières et pour le contrôle des collectivités locales et de leurs attributions foncières. Dans l'État du Delta, la ville de Warri est spécialement touchée à cause des ressources de son soussol. Ijaw, Itsekiri et Urhobo s'y livrent une guerre sans merci et le problème a rejailli sur les multinationales de la région, par exemple lors d'une manifestation qui a fait un mort devant le siège de Mobil en juillet 1998.

Etabli en novembre 1996 à Ogbe-Ijoh, en pays ijaw, le chef-lieu de la collectivité locale de Warri-South a en effet été déménagé pour d'obscures raisons à Ogidigben, en territoire itsekiri. Les troubles qui ont alors démarré ont provoqué la mort de plus de cinq cents personnes en un an, dont une cinquantaine pour le seul mois d'avril 1997. A coup de représailles ijaw, puis itsekiri, en juin et juillet 1997, le cycle des vengeances a bientôt échappé aux tentatives de médiation. Les heurts n'ont pas non plus épargné la campagne environnante, où l'on a recensé quinze victimes en août 1998 et jusqu'à deux cents le 30 mai 1999, lors d'une attaque ijaw sur le village itsekiri d'Arunton, près du terminal pétrolier de Chevron sur la rivière Escravos au sud de Warri. Le conflit a également gagné les Urhobo de l'arrière-pays à l'occasion d'affrontements qui ont fait huit, quatre et vingt morts respectivement le 8 avril, le 31 mai et le 11 juin 1999. Malgré quelques accalmies, le feu a continué de couver. A propos de la délimitation des circonscriptions électorales, cette fois, les violences ont repris en mars 2003 et causé une centaine de morts à l'approche d'un scrutin présidentiel, obligeant les compagnies pétrolières à se retirer pour un temps ${ }^{6}$.

$\mathrm{Du}$ fait de son ancienneté et de sa prééminence au Nigeria, Shell est souvent la première visée par les contestations malgré la concurrence grandissante d'autres multinationales telles que, par ordre décroissant, Mobil, Chevron, TotalFinaElf, Agip et $\mathrm{Texaco}^{7}$. A coups de sabotages de pipe-lines, d'attaques à main armée, d'enlèvements, de lock-out, de grèves et de manifestations, Shell a perdu jusqu'à un quart de sa production au Nigeria en 1999, pendant que Elf enregistrait un déficit supérieur à quatre-vingts journées de travail. L'année suivante, on a observé une relative décrue des agressions, des kidnappings et des demandes de rançons, qui ternissaient l'image de la lutte, mais une augmentation des vols d'équipements et des actes de piratage des conduites ${ }^{8}$.

Ce dernier aspect, qui témoigne d'une autre forme de criminalité, est sans doute le plus difficile à combattre car il met en évidence d'indéniables complicités entre les employés des compagnies exploitantes et les mafias chargées de la revente du pétrole au marché noir. En sous-main, des militaires nigérians fournissent la logistique et les bateaux pour exporter le brut ou l'essence raffinée. Les techniciens du pétrole, eux, aident les pirates en révélant les points névralgiques et le tracé exact des pipe-lines enterrés sous terre ou sous l'eau'. Les sous-traitants - et non les autochtones - sont d'ailleurs les premiers bénéficiaires des travaux qu'entraîne la réparation des dégâts : la redistribution des emplois générés à cette occasion donne lieu à un véritable racket. Le paysan, au contraire, a beaucoup à perdre lors d'une marée noire qui saccage ses cultures pour les années à venir, alors que le bénéfice éventuel d'un contrat de nettoyage ne dure pas plus de quelques mois. De ce point de vue, on ne peut s'en tenir à l'argument des pétroliers selon lequel les communautés locales, poussées par un besoin 
pressant d'argent, seraient les premières responsables de sabotages dont elles n'auraient pas conscience des effets destructeurs à long terme.

La confrontation avec les multinationales a ainsi pris un tour criminel en dépit de ses enjeux politiques. Elle s'est développée autour de trois principales demandes: du travail, des indemnités foncières et des compensations pour la pollution. Ce dernier point a largement retenu l'attention car il a été très médiatisé sous l'impulsion de l'écrivain ogoni Ken Saro-Wiwa, devenu un martyr de la cause après sa pendaison par la junte du général Sani Abacha en 1995. Auparavant, la question du respect de l'environnement ne préoccupait guère; au Nigeria, elle n'avait pas tant visé les compagnies pétrolières que les gouvernements occidentaux venus déverser leurs déchets toxiques en Afrique, ou les militaires qui auraient contaminé la terre en utilisant des armes chimiques lors de la guerre du Biafra. Fort de ses appuis dans les milieux écologistes à l'étranger, Ken Saro-Wiwa, lui, a su articuler les griefs locaux de façon à mobiliser la communauté internationale en sa faveur. Il a notamment dénoncé les pratiques d'une industrie qui ne respectait pas les standards européens et recensait plus d'accidents de travail qu'en mer du Nord, zone pourtant réputée dangereuse au vu de ses aléas climatiques. Dans le delta du Niger, les pétroliers laissaient les torchères de gaz brûler à ciel ouvert et ne se souciaient guère d'enterrer les pipe-lines pour réduire les risques d'explosion ou de fuite. A défaut d'obtenir des compensations, les partisans de Ken Saro-Wiwa ont alors réussi à bloquer la production en pays ogoni et à en chasser les opérateurs, tandis que Shell, poussée par les menaces de boycott et la pression des lobbies « verts » en Occident, s'engageait à cesser la combustion de gaz à ciel ouvert, à remplacer les conduites détériorées et à recycler les eaux usées.

En ce qui concerne le marché du travail, les autochtones ont aussi eu le sentiment d'avoir été lésés du fait que, structurellement, l'industrie pétrolière requiert énormément de capitaux mais suscite peu d'embauche. Shell, par exemple, emploie 3 700 permanents et, indirectement, 6000 sous-traitants. Pour une entreprise qui produit $40 \%$ du pétrole nigérian, lui-même à l'origine de $97 \%$ des rentrées en devises dans le pays, c'est très peu. En comparaison, une compagnie comme Michelin occupe 3000 travailleurs, dont 2000 sur ses plantations d'hévéa de la région de Benin et 1000 dans son usine de pneus à Port Harcourt. Du pétrole aux mines de pierres précieuses, les salaires des industries d'extraction atteignent, il est vrai, des niveaux bien plus élevés, en général, que dans des secteurs manufacturiers tels que le textile, le bâtiment ou l'agroalimentaire. Il n'en reste pas moins que la " générosité » des multinationales, en la matière, a plutôt contribué à accentuer les inégalités sociales et l'agressivité des laissés pour compte du développement.

Les frustrations s'avèrent d'autant plus grandes que, sur le plan foncier, le Land Use Act de 1978 a dépossédé les communautés locales de leurs prérogatives, réduites à un droit d'usage. En vertu de cette loi, édictée sous la forme d'un décret militaire, les gouverneurs ont pu exproprier les autochtones et toucher les compensations que les compagnies pétrolières reversaient autrefois aux chefs et aux propriétaires coutumiers. Résultat, les fonds ont été détournés à d'autres fins, tandis que les compagnies pétrolières n'ont plus négocié des loyers sur la terre mais de simples forfaits visant à compenser les dégâts environnementaux. Fixés arbitrairement par le gouvernement nigérian d'après des taux qui dataient des années 1970, ces forfaits n'ont, de surcroît, pas pris en compte l'inflation et n'ont absolument pas permis de compenser, pour les années à venir, le manque à gagner de la destruction d'un manguier par exemple. 
Aussi les sabotages de pipe-lines se sont-ils multipliés avec l'espoir de récupérer des dommages et intérêts ou de décrocher un emploi dans le cadre des contrats de nettoyage. Les dégâts ont été plus ou moins importants suivant qu'il s'agissait des conduites de la NNPC, qui transportaient de l'essence raffinée, fluide et hautement inflammable, ou des multinationales, qui véhiculaient du brut, lourd et polluant. Le premier type de pipe-line, en l'occurrence, a permis aux pirates de détourner de l'essence à l'usage local ou pour la revente au marché noir mais s'est révélé extrêmement dangereux. Cible de la majorité des attaques, peut-être $80 \%$, le deuxième type de pipe-line, lui, n'a guère laissé de chances aux autochtones d'obtenir des dommages et intérêts. Dans la très grande majorité des cas, en effet, les actions en justice des plaignants n'ont pas abouti car les avocats des compagnies pétrolières ont dégagé les opérateurs de leurs responsabilités en arguant de la criminalité et de la préméditation de l'acte à l'origine de la rupture des flux. Shell prétend ainsi que $40 \%$ des fuites observées ont été causées par des sabotages, ce qui représentait $57 \%$ du volume de pétrole répandu dans la nature en l'an $2000^{10}$.

Les complexes « pétro-sécuritaires "

Une telle agitation a naturellement conduit les multinationales à opérer un repli stratégique dans des «bases-vie» fortifiées. Mais l'explosion de violence n'a pas entraîné un véritable retrait, solution extrême que ne souhaitaient ni les autorités ni les compagnies exploitantes. Le gouvernement fédéral du Nigeria, en particulier, ne pouvait guère se passer d'une manne qui lui procurait la quasi-totalité de ses ressources en devises. Depuis la tentative de sécession du Biafra en 1967, le contrôle de l'État sur le delta du Niger, qui plus est, représentait un enjeu lourd de symboles, le fleuve constituant l'épine dorsale d'un pays auquel il avait donné son nom à partir du mot Niger Area. La communauté internationale, de son côté, ne voulait pas arrêter de commercer avec une région qui fournissait environ $8 \%$ des importations de pétrole en France et aux Etats-Unis. Quant aux compagnies pétrolières, enfin, elles n'avaient pas cessé d'engranger des bénéfices et n'avaient nullement l'intention de renoncer à des gisements qui, malgré les troubles, conservaient des atouts certains. De bonne qualité, très bien coté sur les marchés financiers, le pétrole nigérian a continué d'offrir d'indéniables avantages comparatifs relativement aux gisements de la mer du Nord ou du Golfe de Mexique. Si son coût d'exploitation a toujours été supérieur à ceux des pays du Moyen-Orient, sa prospection est restée une des plus faciles du monde : seulement $5 \%$ des forages n'obtiennent aucun résultat. A meilleure preuve, la persistance d'une situation quasi-insurrectionnelle n'a pas empêché les multinationales d'investir cinq milliards de dollars dans une unité de production de gaz naturel liquéfié à Bonny et la construction d'un pipeline ouest-africain pour ravitailler le Bénin, le Togo et le Ghana en pétrole.

Plutôt que de se retirer, les opérateurs ont donc préféré suivre une stratégie en quatre volets : en délocalisant leurs activités vers l'off-shore, en améliorant leurs politiques de relations publiques, en redistribuant une partie des bénéfices sous la forme de projets de développement, en recourant à la coercition, enfin ${ }^{11}$. Ce dernier aspect, le plus visible du complexe " pétro-sécuritaire ", a reposé sur un triptyque composé à la fois de gardes privés, de forces de l'ordre gouvernementales et de services de surveillance internes aux entreprises, en particulier aux points les plus sensibles de la phase d'exploitation, dont le sabotage pouvait bloquer tout le reste de la production, notamment autour des terminaux pétroliers d'Anten, Bonny, Brass, Escravos, Forcados, Ima, Odudu, Oso Field, Pennigton, Port Harcourt, Qua Iboe et Ukpokiti. 
Très controversé, l'usage de la force publique par des opérateurs économiques a, en quelque sorte, tenu lieu de privatisation, nous obligeant à revenir plus précisément sur le rôle de la corruption lorsqu'il s'agissait d'acheter la complaisance de l'armée ou des chefs traditionnels. Sous la pression sociale, la corruption, en tant que mode de redistribution des richesses, n'est certainement pas qu'une affaire d'enrichissement personnel ${ }^{12}$. Elle entretient de vastes réseaux de pouvoir et traverse tous les pans de la société, ce qui légitime et assure la solidité de l'ensemble de l'édifice. Dans le cadre d'une économie de la rente et de l'extraction, pour ne pas dire de la prédation, le boom pétrolier des années 1970 a beaucoup facilité la prévarication à cet égard. L'argent facile de l'or noir a déstructuré la société nigériane, excité les convoitises, enflammé le banditisme armé, précipité l'évasion des capitaux et dénaturé une économie productive qui, à l'Indépendance, reposait essentiellement sur l'agriculture ${ }^{13}$.

Les pétrodollars ont notamment permis aux dirigeants de mener grand train de vie à une époque où le gouvernement nigérian, par solidarité tiers-mondiste, allait jusqu'à régler les arriérés de salaire des fonctionnaires de l'île de la Grenade! La nature frauduleuse de l'enrichissement rapide de quelques individus n'a évidemment pas échappé aux masses et a entraîné une déliquescence générale de la morale publique ${ }^{14}$. La vénalité des comportements politiques a gagné l'ensemble d'une société qui, de par ses rapports traditionnels à la richesse, était effectivement mal préparée face à un tel bouleversement et qui, plutôt que d'en pleurer, a préféré en rire par le biais de formations satiriques comme le "parti de l'homme de la rue" (le Nigerian Commoners Party, qui refusait l'Indépendance en 1954) ou « des détrousseurs du peuple nigérian » (le Nigerian People's Hustlers Party, qui dénonçait les méfaits de la naira society en 1979).

La corruption en uniforme

Seul l'écran de fumée des secrets défense ou bancaire a en effet permis de penser que les militaires étaient moins susceptibles que les civils de détourner des fonds publics. L'illusion vient en grande partie de ce que l'armée a acquis une réputation de rempart contre les dérives affairistes des politiciens élus lorsque, au sortir de sa victoire sur les sécessionnistes biafrais en 1970, elle s'est présentée comme la seule institution capable de garantir l'unité nationale et l'intégrité territoriale du pays. Par défaut, les déchirements des parlementaires ont alors légitimé son intervention dans la gestion des affaires publiques. Beaucoup ont ainsi récusé le "mythe occidental» d'une démocratie indissociable d'un gouvernement civil et se sont déclarés favorables à un mariage de raison entre le kaki et l'agbada, le treillis des militaires et le boubou des notables ${ }^{15}$. Le premier président du Nigeria à l'Indépendance, Nnamdi Azikiwe, a luimême prôné un tel système de dyarchie. D'autres concevaient de leur côté un projet de "ci-mili-cy» réunissant «civils» et «militaires" dans un même effort de "democracy ${ }^{16}$. Il n'est pas jusqu'à d'éminents représentants de la société civile qui, inquiets de l'incapacité du gouvernement intérimaire d'Ernest Shonekan à calmer les tensions sociales, aient applaudi le putsch du général Sani Abacha en novembre $1993^{17}$. Des milieux progressistes, notamment, ont cru que l'armée pouvait être porteuse d'une révolution sociale s'inspirant de la veine populiste des jeunes officiers qui, sous la conduite du major Chukwuemeka Kaduna Nzeogwu, commirent le premier coup d'État du Nigeria en 1966 ou, dans une moindre mesure, des « ramatistes » qui s'emparèrent du pouvoir avec le général Murtala Ramat Mohamed en 1975.

Pourtant, l'armée n'a pas échappé à la corruption ambiante, y compris en période de combat chez les mutins biafrais pendant la guerre civile ${ }^{18}$ ! Au sein même de 
l'institution, des gradés ont fréquemment détourné jusqu'à la moitié de la paie des soldats, ou bien ils l'ont reversée avec retard pour, entre-temps, la placer sur un compte rémunéré et en retirer un bénéfice personnel ${ }^{19}$. La participation à des manœuvres d'entraînement ou à des opérations de la paix à l'étranger ont donné une ampleur internationale aux malversations des militaires. D'après certains, les interférences nigérianes dans les trafics de drogue à grande échelle ont commencé à l'initiative d'officiers de la marine en stage en Inde au tout début des années $1980^{20}$. En 1999, le général indien à la tête de la Mission des Nations Unies en Sierra Leone, l'UNAMSIL, se plaignait quant à lui de la façon dont les « casques blancs » nigérians négociaient l'exportation et la revente de diamants de contrebande avec les rebelles du Revolutionary United Front.

Sachant par ailleurs les ramifications de l'armée dans les secteurs économiques les plus lucratifs du pays, les militaires ont ainsi disposé d'un appréciable volant financier pour " acheter" le soutien des civils aussi bien que le silence de leurs opposants. Le cas de Ken Saro-Wiwa est éclairant à ce sujet, puisque le général Ibrahim Babangida, surnommé « Maradona » à cause de son habileté à dribbler entre les écueils de la vie politique nigériane, l'a tantôt jeté en prison, tantôt promu directeur du MAMSER, l'organe de propagande du régime. Seuls les idéalistes ont pu voir une forme d'entrisme et d'activisme dans les compromissions du leader ogoni avec la junte ${ }^{21}$. En réalité, les atermoiements de Ken Saro-Wiwa à l'égard de Babangida rentraient dans le cadre de marchandages courants au Nigeria : une sorte de rapport de force qui consiste, pour les opposants, à faire monter les enchères et à obtenir des postes honorifiques en échange d'un ralliement toujours volatil.

De ce point de vue, il paraît d'ailleurs impossible que le président Olusegun Obsanjo, réélu en 2003, renonce à de telles pratiques, à moins "d'assécher" ses clientèles politiques et de réduire sa base sociale jusqu'à perdre le pouvoir par le biais d'une procédure d'impeachment ou d'un putsch. A la fin de l'année 2000, le gouvernement a généreusement "arrosé » les sénateurs afin de résoudre une crise qui opposait les instances exécutives et législatives. Les élections d'avril 2003 ont également été l'occasion de redistribuer à bon escient les prébendes de l'Etat. Olusegun Obsanjo, à dire vrai, n'en est pas à son coup d'essai. C'est lui qui a nationalisé l'industrie pétrolière et créé la NNPC, laissant un «trou » d'environ trente milliards de francs lorsqu'il a rendu le pouvoir aux civils après les élections de 1979 ! En fait de réformes, l'adoption d'une loi contre la corruption et la nomination, en septembre 2000, d'une commission d'enquête présidée par le juge Mustapha Akanbi ont surtout servi de signaux diplomatiques à destination d'une communauté internationale pour qui la «bonne gouvernance démocratique » devait s'accompagner d'une plus grande transparence en matière d'attribution des marchés publics.

Olusegun Obsanjo a su se gagner la sympathie des bailleurs de fonds à cet égard. Sa présence honorifique au sein du lobbyTransparency International a sans doute valu au Nigeria d'être mieux noté et de laisser au Cameroun le titre peu glorieux de champion mondial de la corruption. Pour le reste, la Commission Akanbi n'a guère dépassé le stade des effets d'annonce et n'a pas eu beaucoup plus d'impact que les organisations de la société civile qui avaient déjà entrepris de lutter contre le fléau, comme la League of Bribe Scorners ou la Anti-Bribery and Corruption Society of Nigeria dès les années 1950. Structurellement, le mandat des enquêteurs était très limité par des contraintes d'ordre politique. D'après le ministre de l'Information Jerry Gana, Olusegun Obsanjo ne 
souhaitait pas poursuivre en justice les ministres renvoyés pour cause de malversations, sous prétexte que cela aurait découragé les vocations à des postes de responsabilité publics ${ }^{22}$ ! Surtout, comment envisager sérieusement qu'un gouvernement arrête de financer ses clientèles si cela doit entraîner sa chute?

Soldats en soldes et polices à louer

Evidemment, un tel système a ses revers. Si les militaires au pouvoir ont pu corrompre, c'est aussi que l'armée est corruptible. L'institution y a perdu en efficacité et en discipline. A coups de passe-droits, des officiers ont fini par préférer les responsabilités politiques à des promotions au sein de la hiérarchie militaire ${ }^{23}$. D'une certaine manière, l'armée s'est vendue au plus offrant. La "gauche nigériane » considère ainsi que le régime du général Ibrahim Babangida a servi les intérêts de la bourgeoisie en asservissant les masses et en appliquant le programme d'ajustement structurel de la Banque mondiale en période de crise économique et de contraction des revenus de l'État à la fin des années $1980^{24}$. Cas extrême, le secteur privé a même pu tenter « d'acheter » l'armée! Pour la première fois au Nigeria, la tentative de coup d'État du major Gideon Orkar, en avril 1990, a été montée en dehors des réseaux militaires grâce à l'aide d'un homme d'affaires, Great Ogboru, qui a fourni véhicules, munitions et combattants, recrutés parmi des vétérans de l'armée ${ }^{25} . .$.

A ce titre, soldats et policiers en soldes font partie de la panoplie sécuritaire des opérateurs privés dans une économie politique de la corruption institutionnalisée. Les compagnies pétrolières rémunèrent par exemple les agents affectés à la surveillance de leurs camps et de leur bases-vie. Shell dispose de contingents spéciaux dont la vocation n'est pas sans rappeler le mandat de la gendarmerie de la Niger Royal Company à la fin du XIXème siècle ${ }^{26}$. Outre 2300 gardes privés qui représentent $20 \%$ du total de ses employés, sous-traitants compris, la Shell, en l'occurrence, entretient en permanence quelque 1400 agents de la police nigériane, dont 600 et 400, respectivement, dans les villes de Port Harcourt et Warri en $1995^{27}$. La compagnie ne paie pas seulement des primes qui viennent substantiellement compléter les salaires que l'État nigérian est censé continuer de verser à ses policiers. A un moment, la Shell a voulu se charger ellemême de fournir en armes ces détachements.

Concernant les autres compagnies pétrolières dans la région, il a fallu un veto du Congrès à Washington pour empêcher, en 1999, que les opérateurs américains financent officiellement les forces de l'ordre nigérianes ${ }^{28}$. Ceux-ci n'ont pas pour autant cessé d'appeler la police à la rescousse, voire l'armée dans les cas graves. En pays ijaw, Chevron, notamment, a transporté des troupes de la marine sur sa plate-forme de Parabe pour réprimer des protestations au cours desquelles deux jeunes ont trouvé la mort en mai 1998. Début janvier 1999, à nouveau, Chevron acheminait en bateau et en hélicoptère des soldats qui tuèrent dix-neuf manifestants à Forcados, devant le terminal de Shell, et cinq paysans au Nord de Warri lors de représailles consistant essentiellement à incendier les villages d'Opia et Ikenyan. Fin janvier 2002, la marine intervenait cette fois depuis le terminal des Italiens d'Agip à Brass et faisait quatre morts pour dégager l'accès d'un chantier de la Chinese National Petroeum Corporation à Liama. En août 2002, encore, un bataillon amphibie tuait une femme lors d'une manifestation devant les bureaux de la Shell et de Chevron à Warri.

La délocalisation, une solution limitée

Le dispositif sécuritaire des compagnies pétrolières ne se limite certes pas à la location de la force publique. Le renfermement dans des bases-vie et le repositionnement 
tactique des unités de production ont joué un rôle complémentaire. Les États de la fédération nigériane les plus affectés par les tensions sont ceux dont la majorité des gisements - 75\% dans le Delta, $60 \%$ dans les Rivers ou Bayelsa - se trouve on-shore. Au contraire, les sites les moins touchés par les protestations sont en mer, car plus éloignés et difficiles d'accès ${ }^{29}$. Ainsi, des compagnies ont été relativement épargnées par les attaques de la population pour la simple raison que l'essentiel de leur production - la totalité chez Mobil, bientôt les deux tiers chez Elf - provenait de gisements à bonne distance des côtes - à la différence de Shell, dont les activités étaient surtout terrestres. Résultat, la tendance est aujourd'hui à l'off-shore, ce qui plaide d'ailleurs en faveur de l'Angola, dont la plupart des gisements se situent en zone maritime et échappent donc aux inconvénients de la guerre sur le continent. Des vingtdeux concessions ouvertes à l'exploration au Nigeria en l'an 2000, par exemple, aucune des onze qui se trouvaient sur le continent n'a trouvé preneur alors que les coûts de production étaient deux fois supérieurs en pleine mer.

Concernant les gisements on-shore, qui continuent de représenter $85 \%$ de l'exploitation, il a en revanche fallu composer avec les communautés locales. Se posait là un problème d'interlocuteur qui n'était pas sans rappeler celui du colonisateur britannique essayant d'administrer des sociétés acéphales. Tant les pêcheurs ijaw que les paysans ogoni n'avaient jamais connu d'autorité centrale avant l'arrivée des Européens. Certains villages ijaw étaient vaguement présidés par un Ancien, l'amaokosowei, ou par le descendant du fondateur du lignage, l'amanyanabo; seules quelques communautés avaient réussi, en participant à la traite des esclaves, à constituer de petits empires commerciaux et à s'ériger en cités-États à la fin du XIXème siècle ${ }^{30}$.

Dans un tel contexte culturel et historique, il était très difficile, pour les compagnies pétrolières, de négocier un accord avec une quelconque autorité "traditionnelle " susceptible de maintenir l'ordre et de faire appliquer ses directives sans avoir auparavant obtenu le consensus nécessaire à des prises de décision collectives ${ }^{31}$. Récemment, le PDG de la Shell au Nigeria s'est ainsi plaint de ne pouvoir traiter avec une organisation représentative de l'ensemble de la communauté ogoni ${ }^{32}$. Dominé par les Khana de Ken Saro-Wiwa, le MOSOP (Movement for the Survival of Ogoni People), en l'occurrence, n'a pas su transcender ses divisions claniques et s'est opposé aux Gokana des quatre chefs coutumiers qui, assassinés, servirent de prétexte à la junte du général Sani Abacha pour pendre l'écrivain rebelle en 1995.

De fait, la révolte des régions pétrolifères du Nigeria a, pour beaucoup, transcrit l'exaspération de la jeunesse face à des chefs corrompus qui, jusqu'alors, s'étaient contentés d'empocher les pots-de-vin des multinationales. De façon significative, l'article 18 de la Constitution de l'Ijaw National Congress interdit aujourd'hui aux autorités traditionnelles de vendre la moindre portion de terre. Face à la montée de la contestation, acheter la complicité de quelques notables bien placés ne suffisait plus et les compagnies pétrolières ont dû changer de tactique en étendant leur politique sociale à d'autres types de bénéficiaires. De 1996 à 2000, le budget de Shell consacré à des projets de développement a ainsi doublé et franchi la barre des 60 millions de dollars, tandis que celui de Elf passait de 2 millions de dollars en 1994 à près de 8,5 en 2000. Pareille augmentation a notamment concerné les bourses permettant aux habitants de la région de poursuivre des études : environ 800 par an chez Elf, près de 3 
200 chez Shell, dont plus de 500 en université - au lieu de 6 lorsqu'un tel programme avait débuté en 1953.

Le développement, forme de paternalisme tropical

Les opérateurs ont peaufiné leur stratégie de relations publiques à cet égard. Elf, qui avait inauguré un premier comité consultatif à Obagi en 1973, a renforcé sa politique communautaire ${ }^{33}$. Chevron et Texaco ont, quant à eux, signé un accord régional avec le Programme des Nations Unies pour le développement en 2002. A partir de 1996, Shell a également cherché à faire participer les communautés à des projets qui étaient autrefois imposés sans véritable consultation de la base et qui dépérissaient bien vite. Désormais, $67 \%$ des projets de la sorte seraient en état de fonctionner selon les services de communication de la compagnie. Leur taux de succès et de popularité aurait progressé de $47 \%$ à $62 \%$ d'après des chiffres officiels, à moins qu'il ne plafonne autour de $26 \%$ si l'on en croit des experts indépendants, la catégorie des projets "partiellement réussis " permettant de faire basculer la balance dans un sens ou dans un autre suivant qu'on l'inclut dans la colonne des succès ou des échecs ${ }^{34}$ !

Il faut savoir lire entre les chiffres dans ce domaine, sachant que les compagnies pétrolières refusent l'accès de leurs archives aux chercheurs et ne répondent pas aux questions trop sensibles ${ }^{35}$. Des 60 millions de dollars que Shell dit avoir alloué à des projets de développement en l'an 2000, plus de 33 ont été destinés à la construction de routes qui servaient aussi aux opérations d'exploitation. Autre jeu d'écriture, Shell déclare avoir dépensé plus de 513 millions de dollars en faveur de l'environnement entre 1996 et 2000. Or la majeure partie de cette somme - 282 millions de dollars - a en réalité été consacrée à la canalisation des torchères brûlant à ciel ouvert, qui s'inscrivait dans le cadre du grand chantier de commercialisation du gaz naturel au Nigeria. Le rapport d'Elf, il est vrai, s'avère encore moins explicite. La compagnie française ne signale pas si son budget «social» inclut la construction de routes, n'indique pas le nombre d'accidents du travail, de sabotages ou d'incidents répertoriés, pas plus qu'elle n'a invité un audit externe pour vérifier ses chiffres, à la différence de Shell, qui avait pris le risque de faire appel à une ONG écologiste, Pro-Natura International.

Dans tous les cas, les multinationales du pétrole n'ont, de toutes façons, pas la prétention de jouer aux opérateurs humanitaires ou aux courtiers de la coopération technique intergouvernementale. La finalité de leurs projets de développement est essentiellement d'entretenir des relations de "bon voisinage » avec les communautés sur les territoires desquelles elles exploitent des gisements ${ }^{36}$. Qu'il s'agisse de construire des habitations à loyers modérés, à l'instar des Américains de Gulf Oil à Warri, ou d'ouvrir aux autochtones des cliniques initialement réservées aux employés, la localisation des "œuvres de bienfaisance", toujours à proximité des lieux de production, va dans le sens de la défense d'intérêts bien compris : en faveur de la paix sociale. La question n'est alors pas de savoir si ces projets menacent de rester à l'état "d'éléphants blancs». Avec des préoccupations sécuritaires, la logique du développement, avoue une responsable de Shell, consiste d'abord à calmer les tensions et prévenir les crises afin de ne plus réagir au coup par coup en accordant quelques compensations dans l'urgence, sans rien résoudre des problèmes de fond ${ }^{37}$. Objectif subsidiaire, une telle politique de relations publiques vise également à séduire les journalistes occidentaux, dont certains ont été invités tous frais payés pour entendre 
les opérateurs minimiser leurs responsabilités et présenter les conflits locaux comme la poursuite de vieilles disputes tribales.

Le « syndrome de Monaco »

Résultat, les compagnies pétrolières se sont un peu retrouvées prises à leur propre piège. En effet, la demande de développement restait très grande, y compris de la part des opposants qui dénonçaient l'exploitation du tiers-monde par l'Occident. Les théoriciens de la « dépendance » et du « fordisme périphérique », qui avaient rencontré un écho favorable au Nigeria, imputaient aux multinationales l'essentiel des maux économiques des pays en développement, conférant à celles-ci une dimension politique digne d'un véritable "État derrière l'État ", pour ne pas dire un "État dans l'État " Paradoxalement, de telles thèses ont pu, du même coup, assigner au secteur privé un rôle de complément, voire de substitution à la puissance publique !

Les communautés locales, pour leur part, ont participé de ce constat en dirigeant leur protestation contre le gouvernement nigérian et les compagnies occidentales tout à la fois. Au premier, elles ont réclamé plus d'autonomie et une meilleure redistribution de la manne pétrolière. Des secondes, elles ont non seulement exigé des indemnisations pour avoir dépossédé puis pollué les habitants de la région, mais aussi de réels engagements en matière de développement. Aujourd'hui, les multinationales du pétrole s'occupent de voirie, d'éducation et d'équipements sanitaires. En vingt ans, Shell s'enorgueillit par exemple d'avoir construit 250 écoles, équipé une vingtaine de centres de santé, mis en place une centaine de systèmes d'adduction d'eau et achevé trois centrales au gaz dont les turbines fournissent de l'énergie à l'État de Bayelsa, le seul de la fédération nigériane à ne pas être relié au réseau national d'électricité. Par ailleurs, Shell a récemment étendu ses activités à un secteur bancaire défaillant, sous la forme de micro-crédits qui ont touché 1240 bénéficiaires en l'an 2000, à 98\% des femmes...

Certains considèrent ainsi qu'à force de financer des projets de développement et de fournir des services publics, les multinationales en Afrique ont fini par faire figure de gouvernement privés à l'intérieur d'enclaves autonomes échappant à l'autorité d'États «fantômes ». Le phénomène évoque le "syndrome de Monaco » qui, à travers une apparente souveraineté étatique, voit une portion de territoire placée sous la coupe d'une structure entrepreneuriale et personnelle, en l'occurrence la dynastie des Grimaldi. Sous contrôle vidéo, la principauté monégasque est un des pays les plus surveillés du monde, à raison d'un policier pour 12 nationaux et 75 résidents, contre un pour 235 en France, où ce ratio compte déjà parmi les plus élevés d'Europe. Alors que Monaco recense très peu de crimes violents, une telle omniprésence ne signale pas tant l'efficacité du principe d'un renforcement des forces de l'ordre, mais bien plutôt les mérites très limités d'une répartition des effectifs de police suivant des critères géographique et économique qui consistent à contenir les déviants sur le territoire de la République française et à protéger exclusivement les nantis: rentiers, hommes d'affaires, célébrités du show-biz, joueurs de casinos et dernières familles princières d'une Europe anachronique.

Dans les pays du Sud, ce «syndrome de Monaco " prend certes un tour assez différent, quoique relevant d'une semblable privatisation de la police par la fonction et le territoire. Par la fonction, la corruption et la location des forces de l'ordre détournent les polices de leur mandat, récupéré à des fins personnelles, économiques et politiques, voire criminelles. Par le territoire, la distribution spatiale de l'appareil sécuritaire 
révèle l'existence d'enclaves surprotégées, en particulier lorsque des détachements entiers de police sont spécifiquement affectés à la surveillance d'entités non-étatiques dans des sortes de «zones industrielles renforcées». De fait, les sociétés de gardiennage et les compagnies militaires privées ne constituent certainement pas les seules alternatives de sécurité lorsqu'on analyse de plus près le fonctionnement des établissements industriels ou commerciaux évoluant dans des contextes troubles. Jamais achevé du temps de la colonisation, le monopole virtuel de l'Etat sur l'usage de la violence légitime est toujours à vendre ; l'offre et la demande en déterminent le prix.

\section{NOTES}

1. . Bienen Henry et Fitton Martin, «Soldiers, Politicians and Civil Servants », in PanterBrick, Keith (ed.), Soldiers and Oil : the political transformation of Nigeria, Londres, Frank Cass, 1978, pp. 27-57.

2. . 'Bayo Adekanye J., The retired military as emergent power factor in Nigeria, Ibadan, Heinemann Educational, 1999, pp. 29-67.

3. . Les recommandations de la Banque mondiale ont en effet amené le ministère de la Défense à « externaliser » des services confiés au secteur privé. Voir Dibie Robert A., Public Management And Sustainable Development In Nigeria : Military-Bureaucracy Relationship, Aldershot, Ashgate Publishers, 2002, pp. 266-9.

4. . En 1999, les 46 compagnies de ce type ne constituaient certes qu'une part infime du marché, inférieure à $3 \%$.

5. . Newswatch (Lagos) 30/7/2001, p. 43.

6. . On déplorait à peu près autant de victimes en août suivant.

7. . Frynas Jedrzej Georg, Beck Matthias et Mellahi Kamel, « Maintaining Corporate

Dominance after Decolonization : the 'First Mover Advantage' of Shell-BP in Nigeria ", Review of African Political Economy vol.27, n85, 2000, pp.407-25.

8. . Shell Petroleum Development Company of Nigeria Ltd., 2000 : People and the Environment Annual Report, Lagos, Shell Visual Media Services, 2001, pp.8 et 43.

9. . La technique dite du bunkering, qui affecterait $5 \%$ de la production nigériane, consiste ainsi à siphonner les tuyaux sans les dépressuriser, ce qui permet de ne pas alerter les exploitants. Le détournement des produits pétroliers ne se fait certes pas qu'à partir des oléoducs. La contrebande d'essence fait apparaître une organisation sophistiquée, qui bénéficie de protections au plus haut niveau de l'Etat. A partir des quatre raffineries du Nigeria, nombre de camions citernes n'arrivent jamais à destination et vont revendre leur contenu à meilleur prix dans les pays voisins, qui ne sont pas producteurs et où l'essence n'est pas subventionnée. A l'intérieur du Nigeria, certaines stations services ne sont même pas opérationnelles : structures fantômes, elles n'ont été déclarées aux autorités que pour bénéficier d'un quota d'essence ! Voir Bikam Peter, L'industrie pétrolière et l'aménagement de la distribution de ses produits au Nigeria : impact et perspectives dans l'Est nigérian, Paris, L'Harmattan, 2001, p. 192. 10. . Shell, op. cit., 2001, p. 36. 
11. . Frynas Jedrzej Georg, « Corporate and State Responses to Anti-Oil Protests in the Niger Delta », African Affairs vol.100, $\mathrm{n}^{\circ} 1,2001$, pp. 27-54.

12. . Adeyemi Adedokun, "Corruption in Africa : a case study of Nigeria », in Mushanga, Tibamanya mwene (ed.), Criminology in Africa, Rome, United Nations Interregional Crime and Justice Research Institute, 1992, pp. 83-103.

13. . Walker Ezekiel Ayodele, «Structural Change, the Oil Boom and the Cocoa Economy of Southwestern Nigeria, 1973-1980's », Journal of Modern African Studies vol.38, n¹, mars 2000, pp.71-87. Pour un point de vue plus balancé, voir Guyer Jane, An African Niche Economy. Farming to Feed Ibadan, 1968-1988, Londres, Edinburgh University Press, 1997, 260 p. En relançant la demande alimentaire et en développant le secteur des transports, la manne pétrolière, il est vrai, n'a pas fait que précipiter l'exode rural et vider les campagnes. Elle a aussi enrichi certains paysans, qui se sont achetés des tracteurs ou des engrais.

14. . Barber Karin, « Popular reactions to the Petro-Naira », Journal of Modern African Studies vol.20, $\mathrm{n}^{\circ} 3,1982$, pp. 431-50.

15. . Diamond Larry, « Nigeria in Search of Democracy », Foreign Affairs vol.62, $\mathrm{n}^{\circ} 4$, printemps 1984, p. 912.

16. Nwankwo Arthur A., Civilianized Soldiers : army-civilian government for Nigeria, Enugu, 4th Dimension, 1984, 80 p. Voir aussi Babatope Ebenezer, The Struggle for Democracy in Nigeria, 1960-1993, Lagos, Ebeno Topsy, 1997, p. 79.

17. . Bénéficiant à plein d'un état de grâce, celui-ci a réussi à faire entrer dans son premier gouvernement Babagana Kingibe, le colistier de Moshood Abiola aux élections présidentielles de juin 1993 (annulées par les militaires), Alex Ibru, le patron de presse du groupe Guardian, et Iyorchia Ayu, le président du Sénat dissous ; les portefeuilles du Logement, de la Justice et des Transports, quant à eux, ont été confiés à des Yorouba de l'opposition, respectivement Lateef Jakande, gouverneur de Lagos pendant la Deuxième République, Olu Onagoruwa, un militant des droits de l'homme, et Ebenezer Babatope, un progressiste qui avait soutenu les idées réformistes du général Murtala Mohammed en 1976.

18. . Nwankwo Arthur A., Nigeria : The Challenge of Biafra, Enugu, Fourth Dimension, 1972, 86 p.

19. . Howe Herbert M., Ambiguous order : military forces in African states, Boulder (Co), Lynne Rienner Publishers, 2001, pp. 43, 54, 59 et 158.

20. . Gelbard Robert S., " Drug Trafficking in Southern Africa ", in Rotberg Robert I. et Mills Greg (ed), War and peace in Southern Africa : crime, drugs, armies, and trade, Washington, Brookings Institution Press, 1998, p. 172.

21. . Pegg Scott, « Ken Saro-Wiwa : assessing the multiple legacies of a literary interventionist », Third World Quarterly vol.21, $n^{\circ} 4,2000$, p. 704. Pour une littérature qui encense la figure martyre de Saro-Wiwa, voir Okome Onookome (ed.), Before I am hanged : Ken Saro-Wiwa. Literature, politics, and dissent, Trenton (NJ), Africa World Press, 2000, 224 p. ; McLuckie Craig W. et McPhail Aubrey (ed.), Ken Saro-Wiwa : writer and political activist, Boulder (Co.), Lynne Rienner, 2000, 291 p. ; Ojo-Ade Femi, Ken Saro-wiwa : a bio-critical study, New York, Africana Legacy Press, 1999, 300 p.

22. . Newswatch (Lagos) 30/7/2001, p. 51.

23. . Peters Jimi, The Nigerian military and the state, Londres, Tauris Academic Studies, 1997, pp. 215 et 224.

24. . Nwankwo Arthur, The Military Option to Democracy : Class, Power and Violence in Nigerian Politics, Enugu, 4th Dimension, 1987, 202 p. 
25. . Adekanye J. 'Bayo, " The Military ", in Diamond, Larry Jay, Kirk-Greene Anthony et Oyediran, Oyeley (ed.), Transition Without End : Nigeria Politics and Civil Society Under Babangida, Boulder, L. Rienner, 1997, p. 74.

26. . Baker Geoffrey Leonard, Trade winds on the Niger : the saga of the Royal Niger Company, Londres, Radcliffe Press, 1996, 333 p.

27. . HRW, The Price of Oil. Corporate Responsability and Human Rights Violations in Nigeria's Oil Producing Communities, New York, Human Rights Watch, 1999, p.116 ;

Okonta Ike et Douglas Oronto, Where vultures feast : Shell, human rights, and oil in the Niger Delta, San Francisco, Sierra Club Books, 2001, 267 p.

28. . Ofeimun Odia, « Renewed Nationalist Agitations and the Politics of Subverted Federalism in Nigeria ", in CDHR (ed.), Boiling Point. The Crises in the Oil Producing Communities in Nigeria, Lagos, Committee for the Defence of Human Rights, 2000, p. 69. 29. . En l'occurrence, la proportion de gisements off-shore des autres Etats côtiers variait entre $90 \%$ dans l'Edo ou l'Akwa Ibom, 97\% dans l'Ondo et $100 \%$ à Lagos et dans l'Ogun ou le Cross River. Cf. ND-HERO, Eco-Impacts, Port Harcourt, Niger Delta Human and Environmental Rescue Organisation, vol.1, $\mathrm{n}^{\circ} 2,2001, \mathrm{p} .4$.

30. . Alagoa E.J., « The Ijo Confederacy », in Isawa Elaigwu, J. \& Erim, E.O. (ed.), Foundations of Nigerian Federalism : Precolonial Antecedents, Abuja, National Council on Intergovernmental Relations, vol.1, 1996, pp.112-33.

31. Pareil constat souligne d'ailleurs les limites du clientélisme ethnique, y compris pour l'État nigérian : Dan Etete, le ministre du pétrole du temps de la dictature du général Sani Abacha, était par exemple un Ijaw qui n'a pas réussi à calmer la protestation.

32. . Guardian (Lagos) 25/7/2001, p. 4.

33. . Elf Petroleum Nigeria Ltd., Touching Lives, Lagos, Relations \& Communications Divisions, 2000, p. 7.

34. . LaPin Deirdre, « The Leveraged Buy-in : Creating an Enabling Environment for Business through Strategic Social Investments ", Stavanger (Norvège), Society of Petroleum Engineers International Conference, juin 2000, p. 7. Shell n'a pas autorisé la publication du rapport des consultants invités sur le terrain pour donner une crédibilité scientifique à ses réalisations sociales, nous obligeant à garder l'anonymat de nos sources contradictoires.

35. . Frynas Jedrzej Georg, « Shell in Nigeria : a further contribution », Third World Quarterly vol.21, n¹, 2000, pp. 157-64.

36. . Bolling Landrum Rymer, Private foreign aid : U.S. philanthropy for relief and development, Boulder, Westview Press, 1982, p. 109.

37. . LaPin D., op. cit., 2000.

38. . Pour un ouvrage du genre, voir Biersteker Thomas, Multinationals, the State, and Control of the Nigerian Economy, New Jersey, Princeton University Press, 1987, 344 p. 
INDEX

Mots-clés : conflits, économie politique, entrepreunariat, Militaires, privatisation de la sécurité, sécurité, violence

Index géographique : Afrique de l'ouest, Nigéria 